\title{
Revisión taxonómica del género Bombycilaena (DC.) Smoljan. (Asteraceae)
}

\author{
Santiago Andrés-Sánchez, M. Montserrat Martínez-Ortega \& Enrique Rico
}

\begin{abstract}
ANDRÉS-SÁNCHEZ, S., M. MONTSERRAT MARTÍNEZ-ORTEGA \& E. RICO (2014). Taxonomic revision of the genus Bombycilaena (DC.) Smoljan. (Asteraceae). Candollea 69: 55-63. In Spanish, English and French abstracts.

The first complete taxonomic account of the genus Bombycilaena (DC.) Smoljan. based on the revision of more than 2000 sheets deposited in 28 herbaria is here presented. A detailed description of the genus and a key for the two species therein included are provided, as well as descriptions, habitat preferences, phenology, and geographic distribution. The typification of the name Bombycilaena erecta (L.) Smoljan. is assessed and a neotype is designated for the accepted name Bombycilaena discolor (Pers.) M. Laínz.
\end{abstract}

\section{Key-words}

ASTERACEAE - Bombycilaena - Taxonomy - Typification

\section{Résumé}

ANDRÉS-SÁNCHEZ, S., M. MONTSERRAT MARTÍNEZ-ORTEGA \& E. RICO (2014). Révision taxonomique du genre Bombycilaena (DC.) Smoljan. (Asteraceae). Candollea 69: 55-63. En espagnol, résumés anglais et français.

La première étude taxonomique complète du genre Bombycilaena (DC.) Smoljan., basée sur la révision de plus de 2000 échantillons conservées dans 28 herbiers, est présentée ici. Une description détaillée du genre et une clé d'identification des deux espèces qui y sont incluses sont données, de même que leur description et les données relatives à leur habitat, phénologie et distribution géographique. Le problème lié à la typification de Bombycilaena erecta (L.) Smoljan. est résolu et un néotype est désigné pour le nom Bombycilaena discolor (Pers.) M. Laínz.

Dirección de los autores: SAS: Department of Biological Sciences, HW Pearson Building, University of Cape Town, Private Bag X3, 7701 South Africa; Departamento de Botánica, Facultad de Biología, Universidad de Salamanca, 37007, Salamanca, España. Email: santiandres@usal.es

MMMO: Departamento de Botánica, Facultad de Biología, Universidad de Salamanca, 37007 Salamanca, España.

ER: Departamento de Botánica, Facultad de Biología, Universidad de Salamanca, 37007 Salamanca, España 


\section{Introducción}

El género Bombycilaena (DC.) Smoljan. está integrado por 2 especies, B. discolor (Pers.) Laínz y B. erecta (L.) Smoljan. El grupo posee representación en casi toda Europa, el suroeste de Asia y el norte de África, y ha sido tradicionalmente incluido en la subtribu Filagininae (BENTHAM, 1873) o en el complejo Filago L. (ANDERBERG, 1991), grupo este último taxonómicamente muy complicado, por existir pocos caracteres de utilidad para la delimitación de los géneros y las especies (GALBANY-CASALS \& al., 2010; ANDRÉs-SÁNCHEZ, \& al., 2011; ANDRÉS-SÁNCHEZ \& al., 2013a; ANDRÉs-SÁNCHEZ \& al., 2013b). A pesar de ello, ya desde LinNAEUs (1753) se han reconocido dos grandes grupos. Por un lado, el de Filago y géneros relacionados, y por otro el de Micropus L. y géneros afines. Es en este último en el que se han atribuido tradicionalmente las especies que integran en la actualidad Bombycilaena, unas veces incluidas en el género Micropus (Linnaeus, 1753; CANDOlle, 1836; Willkomm, 1870; BeNTHAM, 1873) y otras segregadas en ese género independiente (Smoljaninova, 1959; Holub, 1975, 1976; Wagenitz, 1980; Devesa, 1987, 2002; ANDERBERG, 1991).

La historia taxonómica del género Bombycilaena ha estado, pues, muy ligada a la de Micropus. CANDolle (1836) fue el primer autor que segregó las especies de este género en una sección diferente dentro del género Micropus (sect. Bombycilaena DC.), grupo que con posterioridad, SMOLJaninova (1959) reconocería como género independiente sobre la base de datos morfológicos. Este tratamiento es seguido también por ANDERBERg (1991), y ha sido respaldado por datos obtenidos a partir de estudios moleculares (GALBANY-CASALS \& al., 2010), si bien los últimos autores, a diferencia del primero, excluyen B. californica (Fisch. \& Mey.) Smoljan., al parecer más relacionada con el resto de géneros norteamericanos del complejo Filago que con Bombycilaena.

De acuerdo con el estudio de SMOLjaninova (1959) y GALBANY-CASALS \& al. (2010), los caracteres morfológicos más destacados para diferenciar Bombycilaena y Micropus son la disposición de las hojas caulinares, alternas en Bombycilaena y opuestas en Micropus, y la morfología de las páleas del receptáculo,con ausencia de apéndices coriáceos en el dorso en Bombycilaena, muy característicos en Micropus.

La interpretación del capítulo en el género no es sencilla. En esta revisión se sigue la interpretación de Holub (1975), MoREFIELD (1992) y GALBANY-CASALS \& al. (2010) para otros géneros del complejo Filago. Así, en Bombycilaena, al igual que en el resto de géneros afines, el involucro está muy reducido y lo constituyen 5 filarios rudimentarios. La estructura que asemeja un involucro la constituyen, en realidad, las páleas del receptáculo, que en el caso de Bombycilaena están muy desarrolladas y se disponen aparentemente en un verticilo. Cada una de ellas encierra una flor femenina, son coriáceas en la fructificación y dehiscente junto con el aquenio.
El número cromosómico encontrado en las especies del género es siempre $2 n=2 x=28$ (HuMPHRIES \& al., 1978; Verlaque \& Filosa, 1993; AndrÉs-SÁnchez \& al., 2013b; WATANABE, 2013), y de al menos B. discolor hay datos sobre el tamaño estimado del genoma $(2 \mathrm{C}=1,4692 \pm 0,005 \mathrm{pg}$; ANDRÉS-SÁNCHEZ \& al., 2013b).

Con estos antecedentes, se ha abordado una revisión del género en el marco del proyecto «Flora iberica» (CASTROVIEJO, 1986-2012), extrapolada a nivel mundial, en la que se trata de delimitar claramente sus especies y resolver los problemas nomenclaturales apuntados por LÓPEZ GONZÁLEZ (1997) y JARVIS (2007), acerca de la confusión en el uso de los nombres de dichas especies.

\section{Materiales y Métodos}

Para el estudio morfológico se ha revisado en material contenido en algo más de 2000 pliegos pertenecientes a 28 herbarios o colecciones (ABH, ALME, BC, BCN, BIO, BM, COA, COI, G, GDA-GDAC, GOET, JACA, JAEN, K, LINN, MA, MACB, MAF, MGC, MJG, MPU, P, PO, SALA, SEV, VAL, W, WU), así como el obtenido tras numerosas recolecciones, sobre todo por, y del que se conserva al menos un pliego testigo en el herbario SALA. De toda la información corológica obtenida sólo se recoge en este trabajo una selección del material estudiado, por lo general tres localidades para cada taxón en cada país donde esté presente, excepto en el caso de la Península Ibérica e Islas Baleares, para las que se detallan hasta una población por provincia. Sobre esta base corológica se han elaborado los mapas de distribución con el software Quantum-GIS 1.8.0.

Para la nomenclatura se han revisado más de 600 obras diferentes, y para la lectotipificación o neotipificación correcta de algunos de los sinónimos taxonómicos se han consultado colecciones históricas de los herbarios BM, G, K, L, LINN, MA, MPU y P.

\section{Resultados y discusión}

Bombycilaena (DC.) Smoljan.

Hierbas anuales, inermes, densamente pelosas, con indumento eglandular, viloso-algodonoso, de grisáceo a grisáceoblanquecino, adpreso. Tallos de erectos a ascendentes, simples o ramificados desde la base o en la inflorescencia, en este caso de forma cimosa a modo de dicasios, pleocasios o monocasios. Hojas del tallo alternas, más o menos adpresas, de linearespatuladas a espatuladas o elípticas, enteras, de obtusas a subobtusas, con un pequeño mucrón escarioso pardusco, de planas a onduladas en el margen, sésiles; hojas involucrantes 3-8, en rosetas que rodean a los glomérulos, de la misma longitud a claramente más largas que estos, de linear-espatuladas a 
espatuladas o elípticas, enteras, de obtusas a subobtusas, con un pequeño mucrón escarioso pardusco, con el margen de plano a ondulado. Inflorescencia en glomérulos de capítulos dispuestos a modo de cimas, bien dicasios, pleocasios o monocasios espiciformes; glomérulos con 2-7 capítulos, subglobosos, densos. Capítulos disciformes, heterógamos, sésiles, globosos, densamente pelosos, con indumento grisáceo, grisblanquecino, blanco, blanco-amarillento o pardo claro. Involucro formado por 5 filarios, de linear-lanceolados a elípticos, de agudos a subobtusos, escariosos en la madurez, verdes con un ancho margen hialino, densamente pelosos en la cara abaxial y glabros en la adaxial. Receptáculo estrecha y levemente cónico. Páleas receptaculares 4-8, imbricadas, dispuestas aparentemente en un verticilo y con un corto pedicelo, erectas en la fructificación, conduplicadas -encierran completamente a una flor femenina en su interior- comprimidas lateralmente, de contorno obovado, estrechadas y asimétricas en la base, agudas, verdes con un pequeño apéndice hialino en el ápice, herbáceas en la floración y coriáceas en la fructificación, densamente pelosas en la cara abaxial y glabras en la adaxial. Flores externas 4-8, femeninas, filiformes, con inserción lateral de la corola, blanquecino-amarillentas con el ápice de rojizo a pardusco, éste ligeramente lacerado; las del centro del capítulo 2-5, hermafroditas, funcionalmente masculinas, tubulares, de blanquecino-amarillentas a verdosas con 4 dientes rojizos. Anteras con dos apéndices basales. Aquenios homomorfos, virguliformes, comprimidos lateralmente, parduscos, glabros. Vilano nulo.

\section{Clave para las especies}

1. Hojas involucrantes planas en el margen, en general de la misma longitud o ligeramente más largas que los glomérulos; glomérulos 7-16 mm de diámetro; capítulos con indumento blanco, blanco-amarillento o pardo claro; páleas del receptáculo 4-5 .......... 1. B. discolor (Pers.) M. Laínz

1a. Hojas involucrantes onduladas en el margen, en general más largas que los glomérulos; glomérulos 3-7(-10) mm de diámetro; capítulos con indumento de grisáceo a grisblanquecino; páleas del receptáculo 5-8

2. B. erecta (L.) Smoljan.

1. Bombycilaena discolor (Pers.) M. Laínz in Bol. Inst. Estud. Asturianos, Supl. Ci. 16: 194. 1973 (fig. 1A-L, 2A).

$\equiv$ Micropus discolor Pers., Syn. P1. 2: 423. 1807. Neotypus (designado aquí): EsPaña. Madrid: San Fernando de Henares, 30TVK5575, $550 \mathrm{~m}$, yesos sobre margas, 18.V.1983, M. Luceño s.n. (MA [448183]!) (fig. 3, flecha).

Hierba anual, densamente pelosa, con indumento de grisáceo a gris-blanquecino. Tallos $2-20 \mathrm{~cm}$, simples o ramificados. Hojas del tallo 3-19 × 0,5-5 mm, planas en el margen, de linear-espatuladas a espatuladas; hojas involucrantes 3-8, de 5-12 $\times 2-4 \mathrm{~mm}$, en general de la misma longitud o ligeramente más largas que los glomérulos, planas en el margen, de linearespatuladas a espatuladas. Glomérulos de 7-16 mm de diámetro, con 2-7 capítulos. Capítulos 2,5-6 × 2-5 mm, en general prácticamente indistinguibles, con indumento blanco, blancoamarillento o pardo claro, de color diferente al del resto de la planta. Filarios 1,5-2,3 × 0,3-1 mm, de agudos a subobtusos, cara abaxial con indumento de densidad igual a la del resto de la planta. Páleas del receptáculo 4-5, de 1,8-3 × 1,5-2 mm. Flores externas 4-5, con corola de 1,5-2 mm; las del centro del capítulo 3-4, con corola de 1,3-1,5 mm. Aquenios 1,5-2 × 0,8$1 \mathrm{~mm}$.

Número cromosómico. $-2 n=28$ (HUMPHRIES \& al., 1978; ANDRÉS-SÁNCHEZ \& al., 2013b).

Hábitat, fenología y distribución. - Pastos de terófitos en márgenes de caminos, barbechos, laderas soleadas, secas y pedregosas, etc., en general basófila; 50-2000 m. III-VII. S de Europa, N de África y SW de Asia (fig. 4).

Iconografías. - VALDÉS \& al. (1987: 31).

Observaciones. - PERSOON (1807) indica en el protólogo de $M$. discolor que el tipo de este taxón es un pliego de Lagasca recolectado cerca de Madrid. Dicho material debería estar depositado en L, donde se conserva el herbario de Persoon, pero allí no existe material relacionado con el binomen que pueda escogerse como lectótipo. Para preservar definitivamente la identidad de $M$. discolor, se ha buscado material recolectado por Lagasca en el herbario del Real Jardín Botánico de Madrid (MA, donde se conserva su herbario), susceptible de ser utilizado para la designación de un neótipo. Sin embargo, aunque se conservan 2 pliegos recolectados por Lagasca y procedentes de Madrid («prope Matritum»), contienen abundante material de las dos especies de Bombycilaena, por lo que se ha descartado su uso en este sentido. Como neótipo se escoge el material del pliego MA [448183], procedente de una recolección reciente de los alrededores de Madrid y que contiene especímenes con la morfología más común y representativa de $B$. discolor.

Material estudiado (selección). - AFGANISTáN: Herb. Griffith, s.d., s.coll. $(\mathrm{K})$.

Algeria: Alger, oeste de Djelfa, norte de Kef Haouas, 17.VI.1954, L. Faurel 161 (MPU); El Bayadh, Dj. Amour, 8-9 km W.S.W. de El Bayadh, 4.VI.1975, Davis 58683 (BM); Constantine, 24.VI.1857, S. Choulette $544(\mathrm{P})$.

Chypre: En mont. Kantara, IV.1880, Sintenis \& Rigo 318 (MPU); Limassol, colinas de Polemidia, 19.V.1883, A. Deflers 559 (MPU).

EsPaña. Álava: Lapuebla de Labarca, 27.V.1983, J. A. Alejandre \& P. M. Uribe-Echebarría s.n. (BIO [704]). Albacete: Barrax, cerro de Don Diego, 18.V.1997, M. B. Crespo \& al. s.n. (ABH [35448]). Alicante: Orihuela, Dehesa de Campoamor, 31.V.1996, S. 


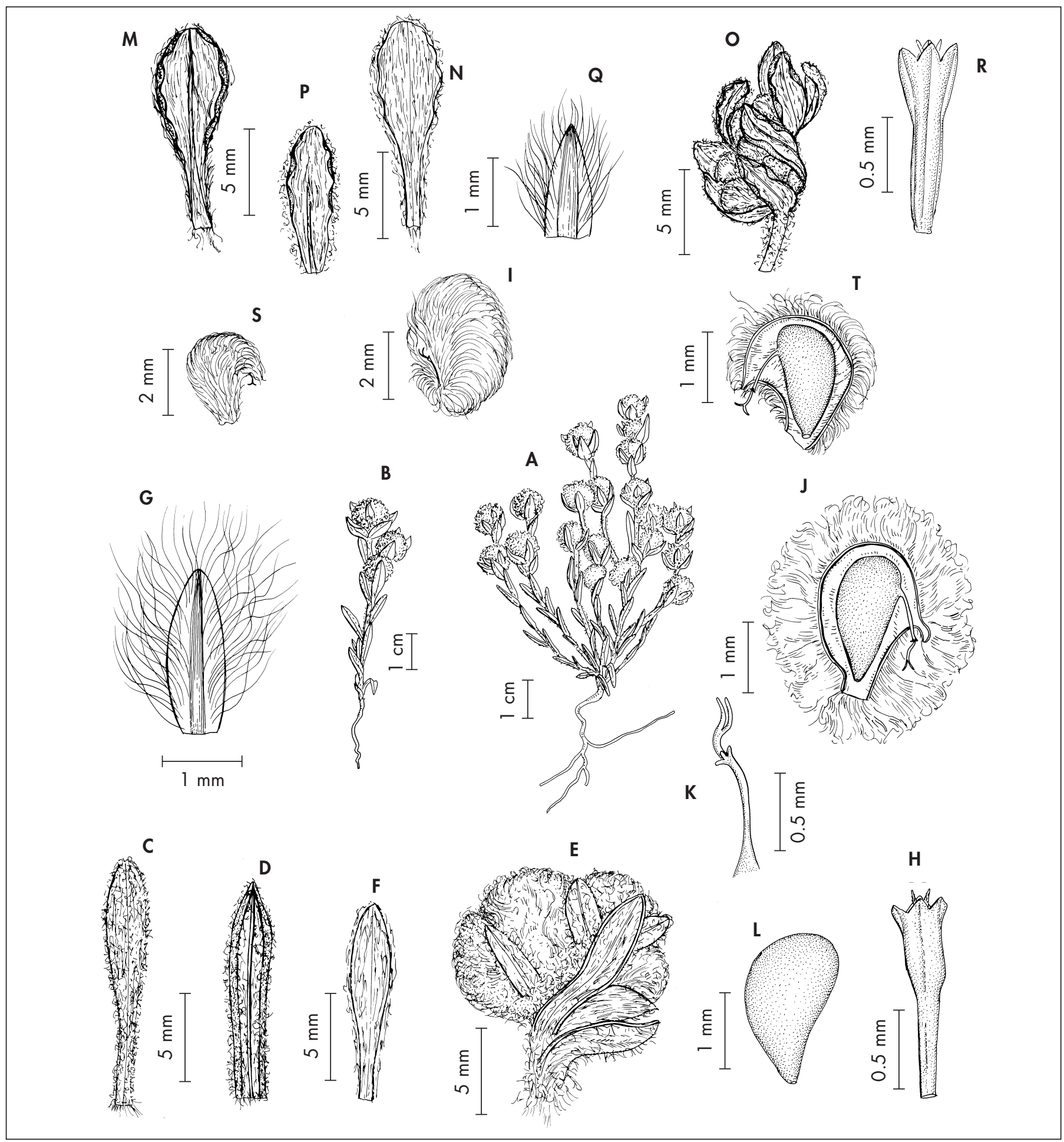

Fig. 1. - Bombycilaena discolor (Pers.) M. Laínz: A-B. Hábito; C. Hoja caulinar, haz; D. Hoja caulinar, envés; E. Glomérulo; F. Hoja involucrante; G. Filario; H. Flor hermafrodita, vista lateral; I. Pálea del receptáculo; J. Sección transversal de la pálea del receptáculo junto con la flor femenina; K. Detalle del estilo y estigmas, detalle; L. Aquenio. Bombycilaena erecta (L.) Smoljan.: M. Hoja caulinar, envés; N. Hoja caulinar, haz; O. Glomérulo; P. Hoja involucrante; Q. Filario; R. Flor hermafrodita, vista lateral; S. Pálea del receptáculo; T. Sección transversal de la pálea del receptáculo junto con la flor femenina.

[A-L: S. Andrés-Sánchez \& al. SA344, SALA; M-T: S. Andrés-Sánchez \& al. SA115, SALA] [Fotographía: S. Andrés-Sánchez] 


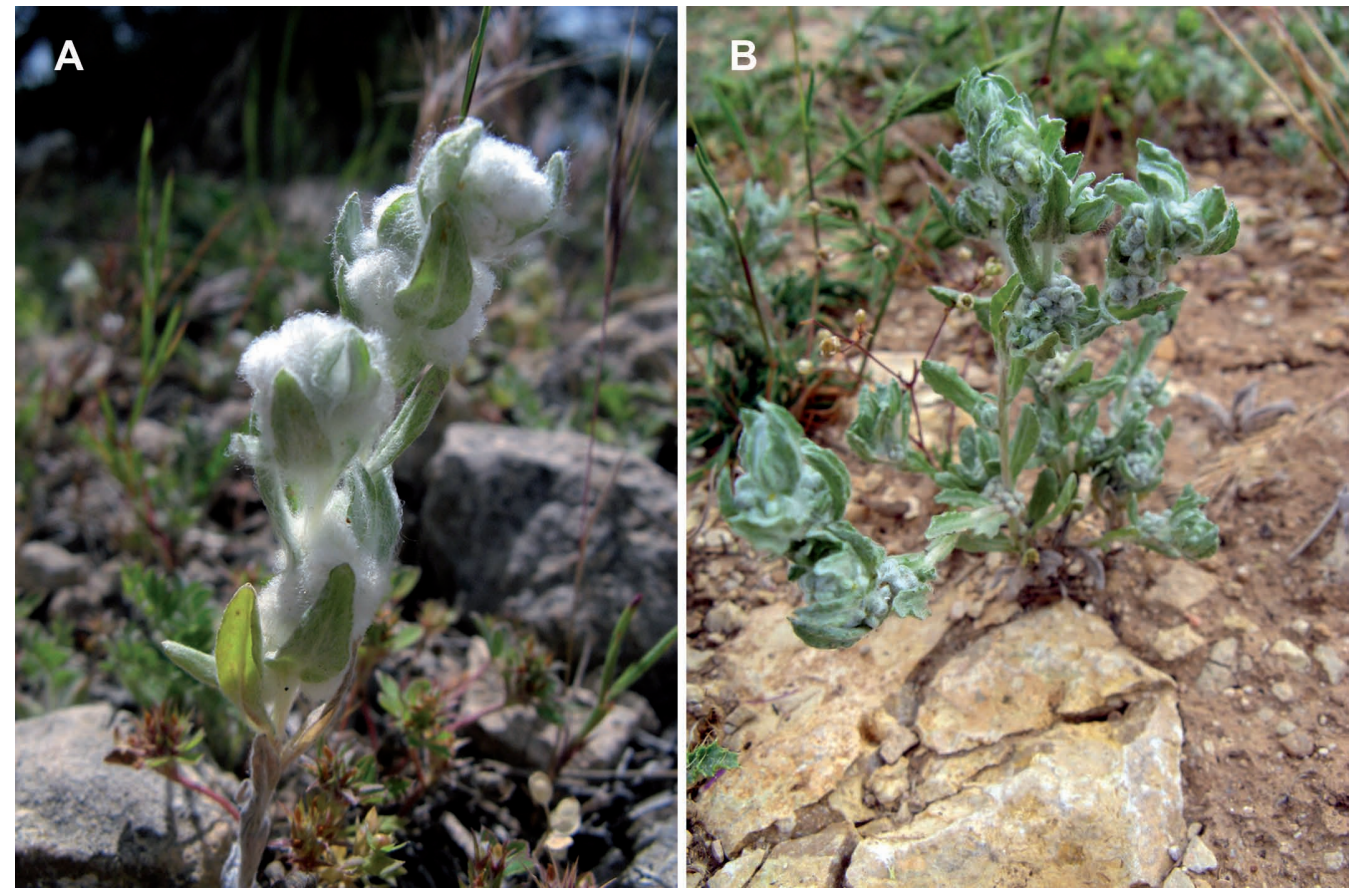

Fig. 2. - Hábitat natural. A. Bombycilaena discolor (Pers.) M. Laínz, Elche de la Sierra, Albacete, España; B. Bombycilaena erecta (L.) Smoljan., Beteta, Cuenca, España. [Fotographía: S. Andrés-Sánchez]

Espinar \& al. s.n. (ABH [31783]). Almería: base de la Sierra de los Filabres, Benizalón, Cortijo de los Pajares, 15.IV.1988, C. Morales \& A. P. Robles s.n. (GDA [29930]). Ávila: cerca de la ciudad de Ávila, 19.VII.1892, A. E. Lomax s.n. (MA [123791]). Badajoz: Maguilla, 21.IV.1984, E. Rico s.n. (BCN [35570]), (SALA [57440]). Burgos: Mena, Salcedo 973 (MA [123773]). Cáceres: Cáceres, finca de La Alberca, 6.IV.1988, M. Ladero \& al. s.n. (SALA [77247]). Cádiz: Olvera, Cortijo de Orihuela, 25.V.1984, A. Aparicio \& J. G. Rowe s.n. (SEV [212928]). Castellón: Peñagolosa, Lagasca s.n. (MA [123786]). Ciudad Real: Alcázar de San Juan, Herencia, 27.V.1973, S. Castroviejo s.n. (MA [203004]). Córdoba: Rute, subida al castillo desde la carretera hacia Carcabuey, 20.V.2010, S. Andrés-Sánchez \& al. SA344 (SALA [139201]). Cuenca: El Pedernoso, 18.V.1968, P. Montserrat s.n. (JACA [111068] [= JACAR22592]). Granada: Cullar de Baza, cercanías de la Venta de Perales, 28.IV.2007, S. Andrés-Sánchez \& al. SA088 (SALA [134275]). Guadalajara: Pareja, 9.VI.1973, A. Segura Zubizarreta s.n. (MA [351763]). Huesca: Candasnos, carretera a Caspe, 28.IV.2001, M. Galbany Casals \& al. s.n. (BCN [3136]). Islas Baleares: Mallorca, Pollença, L. Lloro, 26.V.1889 (P). Jaén: de Ceal a Huesa, 18.IV.2006, S. Andrés-Sánchez \& al. SA061 (SALA [134287]). Lérida: entre Figuerola y Vilamitjana, 16.V.1972, A. Charpin s.n. (G [00035492]). La Rioja: El Rasillo de Cameros, 28.VI, Zubía s.n. (MA [123672]). Madrid: Alcalá de Henares, Universidad, 2.V.1987, F. Lamata s.n. (MA [587584]). Málaga: Istán, Puerto del Lobo, 7.VII.1994, Pérez-
Latorre \& al. s.n. (MGC [39377]). Murcia: Lorca, sierra del Caño, 13.V.1923, Hno. Jerónimo s.n. (MA [123670]). Navarra: Endavia, 28.V.1985, J. A. Alejandre s.n. (MA [339816]). Sevilla: Pruna, S del Tablón, 4.VI.1981, I. Fernández \& B. Valdés s.n. (SEV[212921]). Soria: Añavieja, 2.VI.1973, A. Segura Zubizarreta s.n. (MA [351764]). Tarragona: ribera del Ebro, Flix, 12.VI.1981, J. Molero \& A. Rourá s.n. (BCN [34470]). Toledo: Ontígola, 12.V.1968, P. Montserrat s.n. (JACA [64568][= JACAR22070]). Valencia: Requena, Murviedro, VI.1862, Leresche s.n. (MPU). Valladolid : Tiedra, 8.VI.1981, F. J. Fernández Díez s.n. (SALA [24369]). Zamora: Cañizal, 9.V.2005, M. M. Martinez-Ortega \& S. AndrésSánchez MO1819 (SALA [134225]). Zaragoza: Venta de Sta. Lucia, 2.VII.1971, P. Litzler 71/137E (MPU).

Francia: Charente Inférieure, Chaumes de Sèche-Bec, entre Bords y Saint-Savinien, V/VI.1884, J. Foucaud 873 (P); Yonne, Merry-sur-Yonne, s.d., C. Billot s.n. (P); Hérault, Montpellier, Port Juvenal, V.1857, J. E. Planchon s.n. (MPU).

GreCIA: Monte Kerata, cerca de Eleasin, 23.IV.1891, Heldreich 2234 (K, P); Attica, M. Trikeros, cerca de Eleasin, IV, Heldreich s.n. (K); Macedonia, base del monte Corpiati, 5/17.VII.1857, T. G. Orphanides $773(\mathrm{~K})$.

IRAK: Mesopotamia, s.d., Aucher-Eloy 3127 (K).

Irán: cerca de Shahrud, V.1858, Herbar Bungeanum (P); Mashhad, 1858-1859, Al de Bunge s.n. (P). 


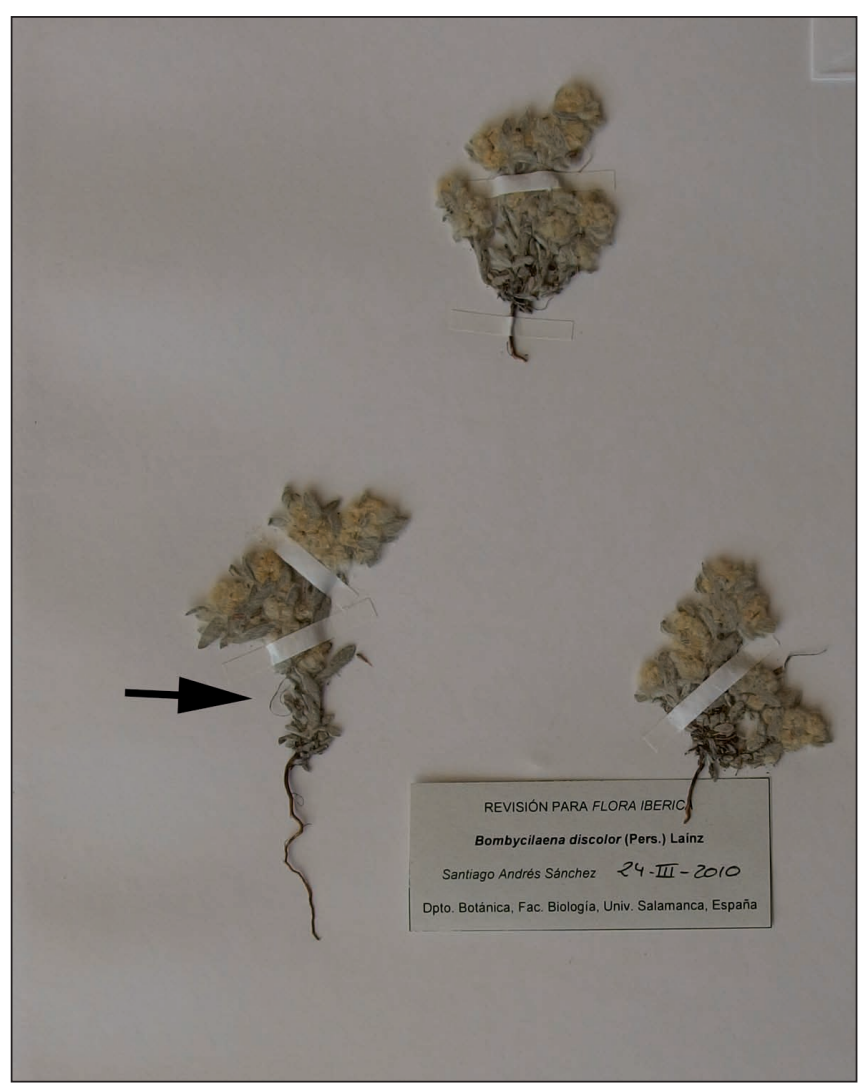

Fig. 3. - Neótipo de Bombycilaena discolor (Pers.) M. Laínz (designado por una flecha).

[M. Luceño s.n., MA] [@ Real Jardín Botánico. Reproducido con autorización]

ItaLIA: Mugello, in m. Giogo Apennini Florentini, 12.VI.1842, De Heldreich s.n. (P).

LiBiA: Tripolitania, SE de Gariara, 1966, C. W. Mitchell 250 (K); Tripolitania, Garian, 13.IV.1933, J. Bornmüller 764 (K); Tripolitania, monte Tekout, 1.V.1938, R. Maire \& M. Weiller 792 (MPU).

Marruecos: Oujda, El-Heimer, 16.IV.2006, S. Andrés-Sánchez \& al. SA038 (SALA [134286]); Meknès-Tafilalet, Midelt, 6.VI.1926, Nain 341 (MPU); Marrakech-Tensift-Al Haouz (Marrakech), Asni (High Atlas), $7 \mathrm{~km}$ por encima de Asni en la pista a Oukaimeden, 14.VI.1974, Reading Univ./ B.M. Exped. (BM).

SIRIA: Aleppo, s.d., Kolscky s.n.(P).

TúNEZ: Gafsa (gobernación), Sened, alrededores del pueblo, 26.III.2009, J. Calvo \& al. JC 3102 (SALA[110224]); Matmata, Kebira, IV.1910, C. J. Pitard 702 (MA[123681]); Medenine, IV.1849, s.d., s.coll. (BM).

Turquia: Smyrne (Izmir), 13.V.1854, B. Balansa s.n. (MPU).
2. Bombycilaena erecta (L.) Smoljan. in Bot. Mater. Gerb. Bot. Inst. Komarova Akad. Nauk. S.S.S.R. 17: 450. 1955 (fig. 1M-T, 2B).

$\equiv$ Micropus erectus L., Sp. Pl. 2: Addenda post indicem. 1753.

Lectotypus (designated by JARVIS, 2007): Habitat in Hispania (LINN[1042.2]!, foto)

Hierba anual, densamente pelosa, con indumento de grisáceo a gris-blanquecino. Tallos 4-22 cm. Hojas del tallo 3-18 $\times 1,2-5,5 \mathrm{~mm}$, elípticas o espatuladas, onduladas en el margen; hojas involucrantes 3-4, de 8-14 × 2-5 mm, en general más largas que los glomérulos, elípticas o espatuladas, onduladas en el margen. Glomérulos de 3-7(-10) mm de diámetro, con 2-6 capítulos. Capítulos 2,5-4 × 2-4 mm, a menudo distinguibles, con indumento de grisáceo a gris-blanquecino, de color similar o apenas diferente al del resto de la planta. Filarios 1,5-1,8 $\times$ 0,4-0,8 $\mathrm{mm}$, agudos, cara abaxial con indumento de densidad similar a la de toda la planta. Páleas del receptáculo 5-8, de 1,5-2,2 $\times 0,8-1,3 \mathrm{~mm}$. Flores externas 5-8, con corola de 1-1,5 mm; las del centro del capítulo 2-5, con corola de 1-1,4 mm. Aquenios 0,8-1,2 x 0,4-0,8 mm.

Número cromosómico. $-2 n=28$ (VerlaQUe \& Filosa, 1993).

Hábitat, fenología y distribución. - Pastos de terófitos en márgenes de caminos, zonas abiertas, barbechos, etc., principalmente en substratos básicos; 0-2000 m. IV-VIII. Europa, desde la Península Ibérica y el Reino Unido hasta Turquía y Rusia, SW de Asia, NW de África (fig. 5).

Iconografías. - FiORI \& PAOLETTI (1933: 442, fig. 3529), SMOLJANinOVa (1955: 451, tab. 29); VALDÉs \& al. (1987: 31).

Observaciones. - Aunque LóPEZ GonZÁLEZ (1997) planteó la necesidad de conservar el nombre $M$. erectus $\mathrm{L}$. con un tipo nuevo, al entender que el tipo de esta especie corresponde a lo que hoy día se conoce como Bombycilaena discolor (una problemática en la que también incide JARVIS, 2007), un nuevo estudio del mismo hace innecesaria que se haga esta propuesta. En efecto, un minucioso estudio del material tipo de esta especie (LINN [1042.2]), a partir de la fotografía en LINN (http://www. linnean-online.org/11003/), permite concluir que el material contenido en dicho pliego corresponde de manera incuestionable a B. erecta (= Micropus erectus), como delatan la longitud de las hojas involucrantes y la ondulación de sus márgenes, el color grisáceo del indumento de los capítulos y también el diámetro de los glomérulos. Sin duda, las nuevas tecnologías han permitido telemáticamente un estudio más detallado del material tipo, y la reconsideración de su identificación, en lo que coincide con nosotros el Dr. López (com. pers.).

Material estudiado (selección). - ALBANIA: Alpes Boreales Albanicae, Bertiscus, 2.VII.1933, Rechinger fil \& Scheffer 54 (K); Illiria, Forbes s.n. (K). 


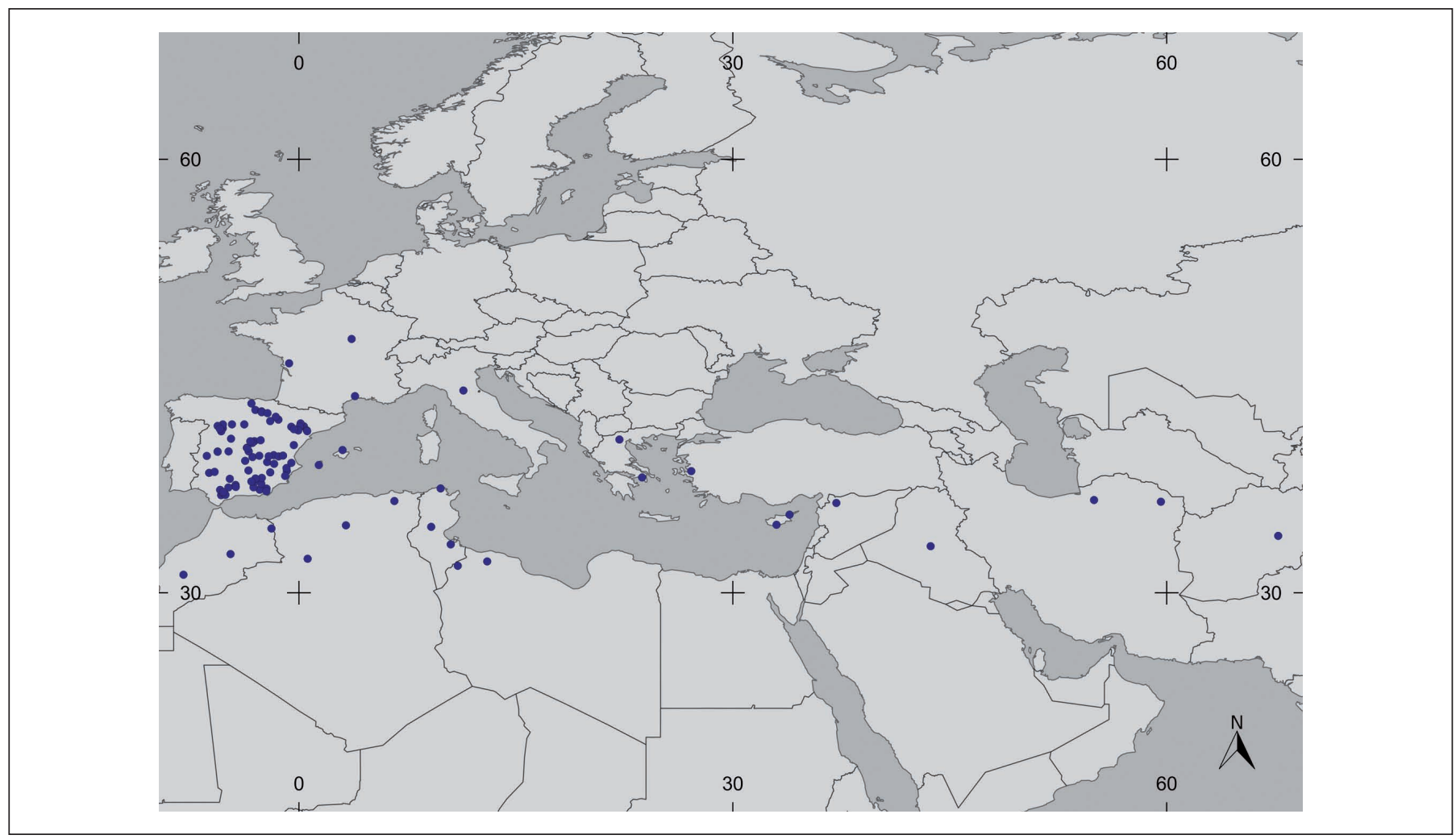

Fig. 4. - Mapa de distribución del material seleccionado de Bombycilaena discolor (Pers.) M. Laínz.

Alemania: Baviera, cerca de W. Neustadt, VII.1840, Schur $1971(\mathrm{P})$.

ANDORRA: Valle de Fontaneda, Coll de Jou, 4.VII.1992, Nieto Feliner \& al. GN3093 (MA[514283]); Parroquia de Sant Julian de Loria, Cova de l'Ossa, 30.V.2004, C. Aedo \& al. 10086 (MA[714840]).

Argelia:. Orán, 20.III.1852, B. Balansa s.n. (MPU).

Armenia: Tavush, entre Hagharatsin y el cruce de la carretera a Chambarak, 21.VI.2005, S. Castroviejo \& al. SC17615 (SALA [128578]).

Austria. Brunn, s.d., Kerner s.n. (P).

Azerbayan: Cerca de Helenendorf, Elisabethopol (Gjandza), V, s.d., F. Hohenacker s.n. (P).

BÉLGICA. Indicada una vez en Bélgica, s.d., Hardy s.n. (P).

Bosnia-Herzegovina. Valle de M canica, 4.VII.1933, B. GilliartSmith $3261(\mathrm{~K})$; in declivitate (SW) monte Laze (Gradina) cerca de Kosovo-Sarajevo, 9.VII.1918, K. Maly s.n. (K).

Bulgaria. Cerca de Varna, 22.V.1907, C. K. Scheneider 223 (K).

Croacia. Dalmatia, Lesina, V-1843, ex legato A. Prior (K); cerca de Fiume, 7.VI.1872, A. M. Smith s.n. (K); Fiume, en pedregales secos, cerca de Wegen, s.d., Noë 1222 (MPU).

EsPaña. Álava: Bernedo, San Román de Campezo, La Muela, 30.VI.1984, G. Morante \& J. A. Alejandre s.n. (MA [399513]). Albacete: Alcaraz, La Molata, 5.VII.1985, A. Pujadas \& E. Hernández s.n. (COA [9317]). Alicante: Puerto de la Carrasqueta, 19.V.1981,
C. Benedi \& J. Molero s.n. (BCN [34483]). Almería: Alhamilla, 7.VI.1967, Ball \& al. s.n. (ALME [3928]). Ávila: cerca de la ciudad de Ávila, 19.VII.1892, A. E. Lomax s.n. (MA [123791]). Barcelona: Bagà (el Berguedá), L’Hospitalet, 5.VI.1983, I. Soriano i Tomás \& al. s.n. (BCN [34598]). Burgos: Cornudilla, Montes Obarenes, pista hacia la Casa del Austriaco, 30.V.2006, L. P. Gavilan \& S. Andrés-Sánchez SA081 (SALA [134313]). Cáceres: Peraleda de la Mata, Las Coscojas, 12.VI.1984, M. Ladero \& al. s.n. (SALA [71317]). Cádiz: El Gastor, Sierra del Gastor, 29.V.1983, A. Aparicio \& al. s.n. (SEV [212927]). Cantabria: Celada, Marlamtes (Enmedio), 6.VII.1987, C. Aedo \& al. s.n. (MA [684879]). Castellón: El Toro, sierra del Toro, 8.VI.2004, M. M. Martínez-Ortega \& al. MO1752 (SALA [134242]). Ciudad Real: Las Aradillas, 18.VI.1935, González Albo \& A. Caballero s.n. (MA [123666]). Córdoba: Morente, 13.VI.1852, E. Bourgeau s.n. (G [00035511]), (P). Cuenca: Beteta, El Tobar, cerro de San Cristóbal, 12.VI.2007, S. Andrés-Sánchez \& al. SA115 (SALA [134270]). Granada: Huescar, sierra de Jureña, 16.VI.04, C. Fernández \& al. s.n. (JAEN [640859]). Guadalajara: Alcolea del Pinar, 7.VII.1977, S. Castroviejo \& E. ValdésBermejo 2378EV (MA [440744]). Huelva: Lepe, El Catalán, 8.VII.1935, González Albo s.n. (MA [449881]). Huesca: Torla, VII.1873, Bordére s.n. (G [00035508]). Islas Baleares: Mallorca, Masanella, cerca de Les Cases de Sa Neu, 30.V.1987, P. Litzler 87/609 Bl (MPU). Jaén: Mancha Real, Sierra Mágina, barranco del Minado, 26-IV-2005, M. M. Martínez-Ortega \& al. MO1800 (SALA [134230]). La Rioja: El Rasillo de Cameros, 26.V, Zubía s.n. (MA [123780]). León: Cremenes, zonas inclinadas terrosas en la parte 


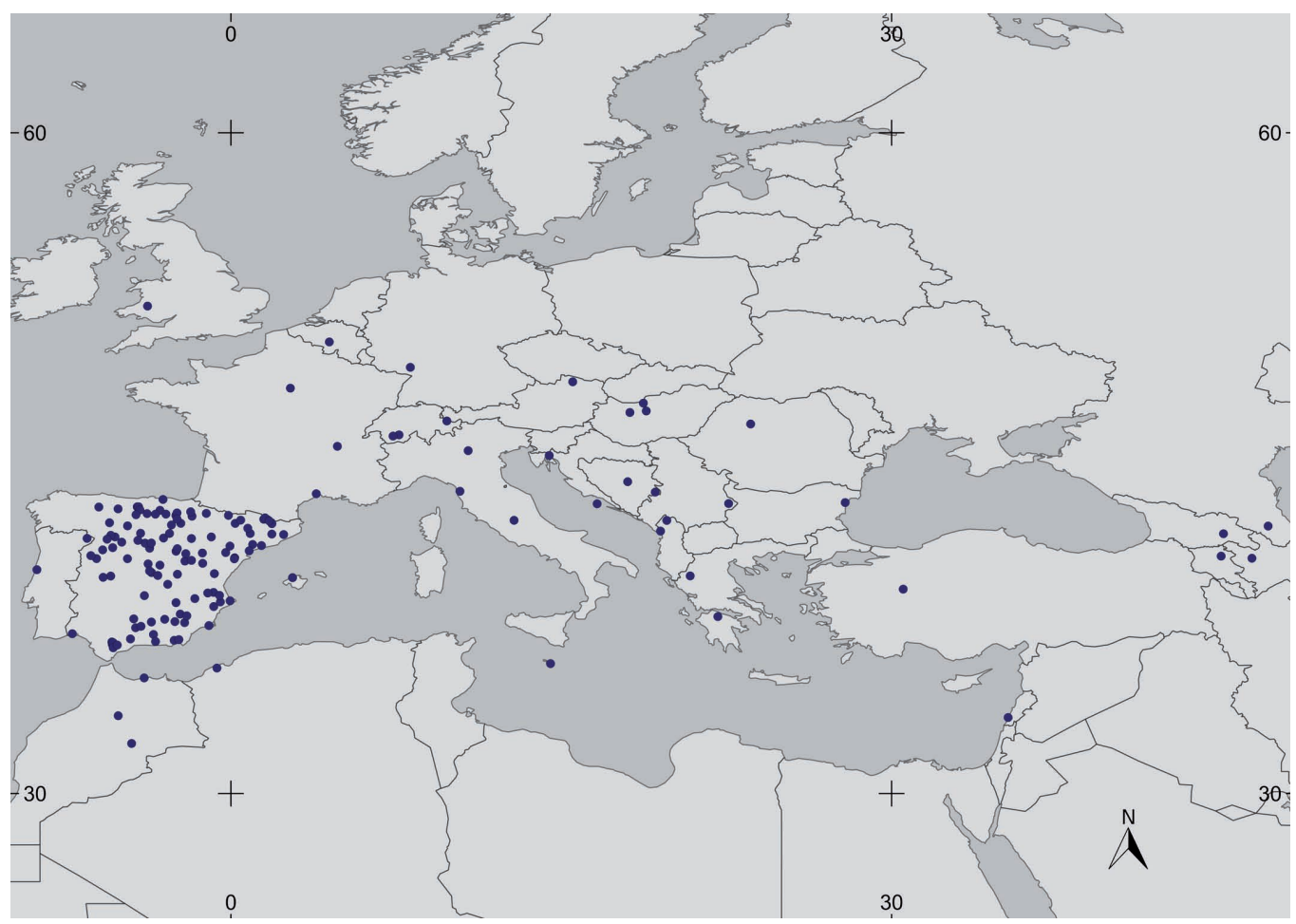

Fig. 5. - Mapa de distribución del material seleccionado de Bombycilaena erecta (L.) Smoljan.

superior del río Esla, 28.VII.1974, J. P. M. Brenan 13074 (K). Lérida: Montsec d'Ares, 25.VI.1916, P. Font Quer s.n. (BC [128485]). Madrid: Olmeda de las Fuentes, 8.VI.2000, J. A. Molina \& P. S. Moreno 080600/3 (MAF [165223]). Málaga: Antequera, Sierra Llana, 14.VI.1930, C. Vicioso s.n. (MA [123749]). Murcia: Cartagena, V.1848, Funk s.n. (K). Navarra: Navascués, alto del Borreguil, 3.VIII.1987, Aizpuru \& Catalán s.n. (MA [704540]). Palencia: Alar del Rey, VII.1950, Losa s.n. (BCN [35568]). Salamanca: Tamames, sierra de las Quilamas, 30.V.1983, E. Rico s.n. (SALA [43667]). Segovia: Grajera, Terradillo, 5.VII.1984, T. Romero s.n. (MA [569049]). Soria: El Burgo de Osma, carretera hacia Almazán, 15.VI.2009, S. Andrés Sánchez \& S. Barrios de León SA308 (SALA [110219]). Tarragona: Serra de Vandellós, 23.IX.1992, J. Molero s.n. (BCN [26340]). Teruel: Cella, 18.VI.2007, L. M. Muñoz Centeno \& A. Abad de Blas s.n. (SALA [135383]). Toledo: Seseña, cerros próximos a El Palomar, 23.V.80, S. Laorga s.n. (MAF [111530]). Valencia: Bicorp, 28.VI.1915, Vicioso s.n. (MA [123792]). Valladolid: Campaspero, 15.VI.1985, T. Romero s.n. (SALA [39677]). Vizcaya: Punta Lucero, 23.VI.1982, M. Onaindia s.n. (MA [704 539]). Zamora: Coreses, 22.V.1983, E. Rico \& A. Guillén s.n. (SALA [43710]). Zaragoza: Aranda de Moncayo, VI.1893, B. Vicioso s.n. (MA [123793]).

Francia: Alrededores de Paris, Fontainebleau, A. Jussieu s.n. (P); Ródano, Lyon, A. Jordan s.n. (P); Hérault, alrededores de Montpellier, garrigues de Bel Avi, 27.V.1966, G. Blanchet s.n. (MPU).

GeorgIA: Kass Oosts Olty, 6.VII.1903, E. Kenun \& E. Koenig s.n. (MPU).
GrecIA: PEL, Ahaia, Kalavrita, Koto Lousoi, 24.VI.2007, C. Navarro \& al. CN7000 (SALA [141008]); IPI, Ioánnina, Flabourari, 29.VI.2007, F. Cabezas \& al. FJC738 (SALA [110221]); Macedonia, base del monte Corfierti, 5/17.VII.1857, T. G. Orphanides 773 (P).

Hungría: Dorog, en Argonium Fr Granf, VI.1878, I. Grundl s.n. (P); en las llanuras de «Rekas», J. A. Tauscher s.n. (MPU); cerca del lago Tarnok, 19.VI.1877, J. A. Tauscher s.n. (MPU).

ITALIA: Monte Baldo, S de Spiazzi, 26.VI.1975, K. Weimer s.n. (MJG [I 821]); Colateo, Apeninos centrales, cerca de Rieti, Colateo, 17.VI.1984, A. Pujadas s.n. (COA [39937]); Pisa, Van Heurck s.n. (K).

LíbANo: Sarepta (Sarafand), 1821, Fischer s.n. (P).

MALTA: La Valette, 7.V.1840, (MPU).

MARruecos: Fez-Boulemane, Ifrane, carretera de Ifrane a Boulemane, $11 \mathrm{~km}$ de Boulemane, 10.VI.1995, M. A. Mateos 6792/95 (SEV [140353]); Taza-Al Hoceima-Taounate, Al-Hoceima, V.1908, M. Gandoger s.n. (G [00035505]); cerca de Rich, Vallée du Ziz, IV.1927, H. Humbert s.n. (MPU).

Portugal. Beira Litoral: Figueira da Foz, Serra da Boa Viagem, Bandeira, 20.VII.1966, J. Matos \& A. Dinis s.n. (COI [0002 0380]); Tras-os-Montes: Bragança, Vimioso, Caçarelos, Minas de Santo Abalde, 14.VI.2010, R. Morales et al. RM2352 (SALA [142154]).

Reino Unido: Walles, S. Léonard, s.d., A. Christ s.n. (K).

RuManía: Cluj, VI.1896, Petry s.n. (MPU). 
RuSIA: Daghestan, s.d., Becker 74 (P).

Serbia: Pirot hacia Slavinja, 21.VII.2010, S. Andrés-Sánchez \& al. SA401 (SALA [110220]).

SuIzA: Valais, Vallée de Saas, VII/VIII.66, Thomas s.n. (K); Valais, Sion, VII.1850, P. Daenen s.n. (P); Valais \& Alpes Lémaniennes, Loèche-Ville, 4.VIII.1909, L. Marret 217 (MPU).

TurQuía: Phrygie, 14.VI.1857, B. Balansa 1274 (MPU).

\section{Agradecimientos}

Este trabajo se encuadra dentro del proyecto "Flora iberica", y ha sido financiado por el Ministerio de Ciencia e Innovación mediante los proyectos CGL2005-05471-C04-03, CGL2008-02982-C03-02/CLI, CGL2011-28613-C03-03, y CGL2012-32574. El primer autor ha sido beneficiario de una beca de investigación (referencia BES-2006-11909) concedida por el Ministerio de Ciencia e Investigación. Por otra parte, queremos mostrar nuestro agradecimiento al Dr. G. López González por su gran ayuda a la hora de resolver la tipificación de Bombycilaena erecta y a J. Castillo por la minuciosa elaboración de la Figura 2, la cual ha sido amablemente cedida por «Flora iberica». También, queremos dar las gracias a los conservadores de los herbarios mencionados en Materiales y métodos, que tan amablemente nos han prestado el material. Por último damos las gracias al revisor anónimo por la profunda revisión de este artículo.

\section{Referencias}

ANDERBERG, A.A. (1991). Taxonomy and phylogeny of the tribe Gnaphalieae (Asteraceae). Opera Bot. 104.

Andrés-SÁnchez, S., M. Galbany-Casals, E. Rico \& M. M. MarTíNEZ-ORTEGA (2011). A nomenclatural treatment for Logfia Cass. and Filago L. (Asteraceae) as newly circumscribed: typification of several names. Taxon 60: 572-576.

Andrés-SÁnchez, S., M. M. MArtínez-Ortega \& E. Rico (2013a). Taxonomic revision of the genus Logfia (Asteraceae, Gnaphalieae) in the Mediterranean region. Anales Jard. Bot. Madrid 70: 7-18.

Andrés-SÁnchez, S., E. Temsch, E. Rico \& M. M. MartínezOrtega (2013b). Genome size in Filago L. (Asteraceae, Gnaphalieae) and related genera: Phylogenetic, evolutionary and ecological implications. Pl. Syst. Evol. 299: 331-345.

Bentham, G. (1873). Tribus IV Inuloideae. In: Bentham, G. \& J. D. HOOKER (ed.), Gen. Pl. 2(1): 180-318. London.

Candolle, A.-P. DE (1836). Micropus L. In Candolle, A.-P. DE \& A. de Candolle (ed.), Prodr. Syst. Nat. Reg. Veg. 5: 460-461. Paris.

CAstroviejo, S. (ed.) (1986-2012). Fl. Iber. CSIC, Madrid.

Devesa, J. (1987). Filago, Logfia, Evax \& Bombycilaena. In: VALDÉs, B., S. TAlaVera \& E. Fernández-Galiano (ed.), Fl. Vasc. Andalucía Occid. 3: 24-31. Ketores editora SA.
Devesa, J. (2002). Bombycilaena, Evacidium, Evax, Filago \& Logfia. In: VAldés, B., M. Rejdali, A. AchHal El KadmiRi, S. L. Jury \& J. M. Montserrat (ed.), Catalogue des plantes vasculaires du nord du Maroc 2: 640-646. CSIC, Madrid.

Fiori, A. \& G. Paoletti (1933) Micropus erectus. In: Fiori, A. \& G. PAOletti (ed.), Iconografia Florae Italicae ossia Flora Italiana Illustrata. Ed. 3 : Firenze.

Galbany-Casals, M., S. Andrés-Sánchez, N. Garcia-Jacas, A. Susanna, E. Rico \& M. M. Martínez-Ortega (2010). How many of Cassini anagrams should there be? Molecular systematics and phylogenetic relationships in the «Filago group» (Asteraceae, Gnaphalieae), with special focus on the genus Filago. Taxon 59: 1671-1689.

Holub, J. (1975). Filago, Logfia, Evax \& Bombycilaena. In: DAvis, P. H. (ed.), Fl. Turkey 5: 101-114. Edinburgh University Press.

Holub, J. (1976). Filago, Logfia, Evax \& Bombycilaena. In: Tutin, T.G., V. H. Heywood, N. A. Burges, D. M. Moore, D. H. Valentine, S. M. Walters \& D. A. WebB (ed.), Fl. Eur. 4: 121125. Cambridge University Press.

Humphries C. J., B. G. Murray, G. Bocquet \& K. Vasudekan (1978). Chromosome numbers of phanerogams from Morocco and Algeria. Bot. Not. 131: 391-406.

JARVIS, C. E. (2007). Order out of Chaos: Linnean plant names and their types. Linnean Society of London, Natural History Museum, London.

Linnaeus, C. (1753). Species Plantarum. Holmiae.

LópEz GonZÁLEZ (1997). Sobre la identidad de Micropus erectus L., basiónimo de Bombycilaena erecta (L.) Smoljan. (Compositae). Anales Jard. Bot. Madrid 55: 480.

MoRefield, J. D. (1992). Resurrection and revision of Hesperevax (Asteraceae: Inuleae). Syst. Bot. 17: 293-310.

Persoon, C. H. (1807). Micropus. In: Persoon, C. H. (ed.), Syn. Pl. 2: 423. Paris.

Smoljaninova, L. (1955) De genere Micropus L. Notulae Systematicae. Bot. Mater. Gerb. Bot. Inst. Komarova Akad. Nauk S.S.S.R. 17: $447-454$

Smoljaninova, L. (1959). Filagininae. In: Schischkin, B. K. (ed.), Fl. USSR 25: 281-303. Science Publishers.

VAldÉs, B., S. TAlaVera \& E. FernándeZ-Galiano (ed.) (1987), Fl. Vasc. Andalucía Occid. 3: 31. Ketores editora SA.

Verlaque, R. \& D. Filosa (1993). Mediterranean chromosome number reports 3 (228-233). Fl. Medit. 3: 364-367.

WAgenitz, G. (1980). Bombycilaena \& Filago. In: Rechinger, K. H. (ed.), Fl. Iran. 4: 11-27. Akademische Druck- u. Verlagsanstalt.

WatAnABE, K. (2013). Index to Chromosome numbers in Asteraceae [http://www.lib.kobe-u.ac.jp/infolib/meta_pub/G0000003 asteraceae_e].

Willkomm, M. (1870). Trib. VII. Gnaphalioideae. In: Willkomm, M. \& J. Lange (ed.), Prodr. Fl. Hispan. 2: 51-67. Stuttgart. 